\title{
A Decomposition-based Large-scale Multi-modal Multi-objective Optimization Algorithm
}

\author{
Yiming Peng, Hisao Ishibuchi ${ }^{\dagger}$ \\ Guangdong Provincial Key Laboratory of Brain-inspired Intelligent Computation, \\ Department of Computer Science and Engineering, Southern University of Science and Technology, Shenzhen 518055, China \\ 11510035@mail.sustech.edu.cn, hisao@sustech.edu.cn
}

\begin{abstract}
A multi-modal multi-objective optimization problem is a special kind of multi-objective optimization problem with multiple Pareto subsets. In this paper, we propose an efficient multi-modal multi-objective optimization algorithm based on the widely used MOEA/D algorithm. In our proposed algorithm, each weight vector has its own sub-population. With a clearing mechanism and a greedy removal strategy, our proposed algorithm can effectively preserve equivalent Pareto optimal solutions (i.e., different Pareto optimal solutions with same objective values). Experimental results show that our proposed algorithm can effectively preserve the diversity of solutions in the decision space when handling large-scale multi-modal multi-objective optimization problems.
\end{abstract}

Keywords-Evolutionary multi-objective optimization; multimodal multi-objective optimization; diversity maintenance; MOEA/D

\section{INTRODUCTION}

A multi-objective optimization problem (MOP) is an optimization problem which has multiple objective functions. Usually, these objective functions are conflicting and cannot be optimized simultaneously. For convenience, all objective functions should be converted into minimization functions. The following equation formulates an MOP without constraints:

$$
\min \boldsymbol{F}(\boldsymbol{x})=\left(f_{1}(\boldsymbol{x}), \ldots, f_{M}(\boldsymbol{x})\right)^{T},
$$

where $\boldsymbol{x}$ is a $D$-dimensional decision vector, and $\boldsymbol{F}$ is a mapping from a $D$-dimensional domain $\Omega$ to an $M$-dimensional range $\mathbb{R}^{M}$.

In the past three decades, researchers have developed a variety of multi-objective evolutionary algorithms (MOEAs). For example, the well-known NSGA-II [1] and MOEA/D [2] can efficiently solve various types of MOPs.

In this paper, we mainly focus on a special type of MOPs called multi-modal multi-objective optimization problems (MMOPs). In MMOPs, the function $\boldsymbol{F}$ in Eq. (1) can be a many-to-one mapping from $\Omega$ to $\mathbb{R}^{M}$. That is, for a

This work was supported by Guangdong Provincial Key Laboratory (Grant No. 2020B121201001), the Program for Guangdong Introducing Innovative and Enterpreneurial Teams (Grant No. 2017ZT07X386), Shenzhen Science and Technology Program (Grant No. KQTD2016112514355531), the Program for University Key Laboratory of Guangdong Province (Grant No. 2017KSYS008).

†Corresponding author: Hisao Ishibuchi, hisao@sustech.edu.cn

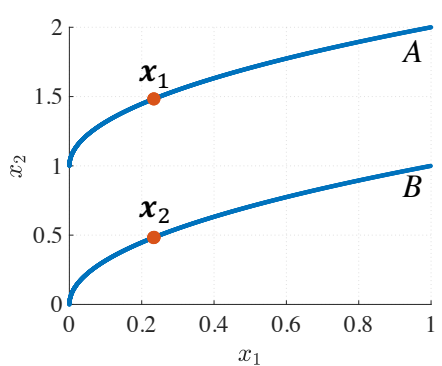

(a) Decision space.

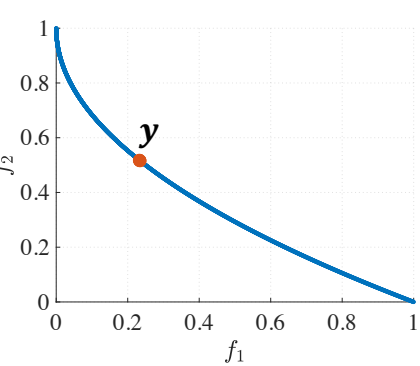

(b) Objective space.
Fig. 1. The two equivalent Pareto subsets ( $A$ and $B$ in (a)) and the Pareto front (the blue curve in (b)) of the SUF3 test problem. Solution $\boldsymbol{y}$ has two inverse images $\boldsymbol{x}_{1}$ and $\boldsymbol{x}_{2}$ in the decision space.

Pareto optimal solution in the objective space, there may exist multiple inverse images in the decision space. Formally, solutions $\boldsymbol{x}_{1}$ and $\boldsymbol{x}_{2}$ are equivalent iff $\boldsymbol{F}\left(\boldsymbol{x}_{1}\right)=\boldsymbol{F}\left(\boldsymbol{x}_{2}\right)$ and $\boldsymbol{x}_{1} \neq \boldsymbol{x}_{2}$. As reported in [3], local optimal solutions is not well-defined for MOPs. Therefore, we only consider global Pareto optimal solutions. Due to the existence of equivalent solutions, an MMOP may have multiple equivalent Pareto subsets, each of which is mapped to the whole Pareto front. For example, in Fig. 1, the SUF3 test problem [4] has two equivalent Pareto subsets $A$ and $B$ and each of them is mapped to the whole Pareto front in the objective space. When handling MMOPs, all equivalent Pareto subsets should be covered. A wide variety of real-world problems are MMOPs. For instance, the space mission design problems [5] and the multi-objective knapsack problems [6] are MMOPs. Solving MMOPs is very useful since equivalent Pareto optimal solutions offer more alternatives for the decision maker [7].

As pointed out in the literature [4], [8], MOEAs cannot efficiently solve MMOPs since they do not consider the diversity of solutions in the decision space. Therefore, several multi-modal multi-objective optimization evolutionary algorithms (MMEAs) are proposed such as DNEA [9], MO_Ring_PSO_SCD [10] and MOEA/D-AD [8]. As reported in [11], these algorithms perform well on MMOPs with lowdimensional decision spaces. However, only a few of them 


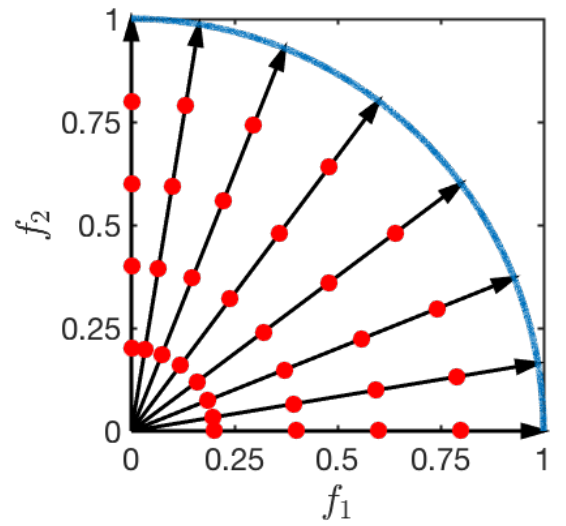

Fig. 2. Explanation of MOEA/D based on the sub-population framework.

can obtain a diverse solution set in the decision space when handling MMOPs with high-dimensional decision spaces (i.e., large-scale MMOPs). To alleviate this issue, we propose an efficient algorithm for large-scale MMOPs.

The rest of the paper is organized as follows. We briefly review related studies on decomposition-based MOEAs and MMEAs in Section [II. In Section III, the proposed MMEA is outlined. Then we compare the proposed MMEA with representative state-of-the-art algorithms on several MMOPs in Section IV] Finally, we summarize the paper and suggest some future research topics in Section $\mathrm{V}$

\section{RELATED WORK}

\section{A. Decomposition-based MOEAs}

In 2007, Zhang and Li proposed MOEA/D [2], the first decomposition-based MOEA. MOEA/D convert an MOP into a set of scalar optimization problems. For this reason, MOEA/D can maintain a strong search ability when handling MOPs with many objectives (i.e., many-objective optimization problems). In addition, MOEA/D is capable of obtaining a uniformly distributed solution set if the given weight vectors uniformly intersect with the Pareto front. Several efficient methods such as MOEA/D-AWA [12] are proposed for the adaptive adjustment of weight vectors.

\section{B. MOEA/D variants based on the sub-population framework}

Although MOEA/D shows promising performance on various types of MOPs, it is not suitable for solving MMOPs as reported in [8]. The main reason is that only one solution is assigned to each weight vector which guides a search direction to a Pareto optimal solution on the Pareto front. Thus, MOEA/D cannot preserve multiple equivalent solutions, i.e., is unable to solve MMOPs. A very straightforward remedy is to assign multiple solutions to each weight vector. In this way, each weight vector is assigned a sub-population instead of a single solution. In Fig. 2, each weight vector has a sub-population containing four solutions. Solutions in the same sub-population search toward equivalent Pareto optimal solutions. To our best knowledge, three MOEA/D variants use this sub-population framework: MOEA/D-AD [8], MOEA/D$\mathrm{M} 2 \mathrm{M}$ [13], and an algorithm from [14]. MOEA/D-M2M is a standard MOEA while the other two algorithms are MMEAs. MOEA/D-M2M lacks a mechanism to preserve equivalent Pareto optimal solutions, which means that it is not suitable for solving MMOPs. Here we briefly introduce MOEA/D-AD and the algorithm from [14].

In MOEA/D-AD, the sub-population size of each weight vector changes adaptively. The mechanism of MOEA/D-AD can be briefly described as follows. In each iteration,

1) An offspring $\boldsymbol{y}$ is generated and assigned to the closest weight vector $\boldsymbol{w}_{i}$ in the objective space. The subpopulation of $\boldsymbol{w}_{i}$ is denoted as $P_{i}$

2) The closest $L$ solutions (denoted as $Q$ ) to $\boldsymbol{y}$ in the decision space are selected from the whole population.

3) The offspring $\boldsymbol{y}$ will be added to $P_{i}$ if one of the following two conditions is met:

- If $P_{i} \cap Q \neq \emptyset$, and at least one solution in $P_{i} \cap$ $Q$ has the worse scalarizing function value than $\boldsymbol{y}$. Solutions worse than $\boldsymbol{y}$ will be removed from $P_{i}$

- If $P_{i} \cap Q=\emptyset$.

The niching structure in the decision space is maintained by the procedures described in step 3). The offspring $\boldsymbol{y}$ only competes with solutions in the current sub-population that are its neighbors in the decision space. In this way, the subpopulation of a weight vector can keep multiple equivalent solutions. Since MOEA/D-AD uses an unbounded population, the size of the whole population may become very large after many iterations.

In the algorithm from [14], each weight vector is assigned $k$ solutions. In this way, a population is separated into $k$ grids, each of which contains one solution from each weight vector. For each grid, the fitness of a solution $\boldsymbol{x}$ which is assigned to a weight vector $\boldsymbol{w}$ is evaluated based on the following equation:

$$
f(\boldsymbol{x})=w_{1} g(\boldsymbol{w}, \boldsymbol{x})+w_{2} d_{m i n}+w_{3} d_{\text {avg }},
$$

where $g$ is an aggregation function, $d_{\min }$ is the minimum Euclidean distance from $\boldsymbol{x}$ to other solutions assigned to $\boldsymbol{w}$, and $d_{a v g}$ is the average Euclidean distance from $\boldsymbol{x}$ to solutions assigned to other weight vectors. These three functions are composed using weights $w_{1}, w_{2}$ and $w_{3}$.

\section{Proposed Algorithm}

\section{A. Basic ideas}

Let us consider solving an MMOP with $k$ equivalent Pareto subsets. Suppose that a weight vector $\boldsymbol{w}$ intersects with the Pareto front of the given MMOP at $\boldsymbol{p}^{*}$. As shown in Eq. (3), we can find at most $k$ different Pareto optimal solutions $\left\{\boldsymbol{x}_{1}^{*}, \boldsymbol{x}_{2}^{*} \ldots \boldsymbol{x}_{k}^{*}\right\}$ in the decision space that are corresponding to $\boldsymbol{p}^{*}$. In addition, the scalarizing function values $g$ (with respect to $\boldsymbol{w}$ ) of these $k$ solutions are also equal to the minimum value (i.e., 0).

$$
\begin{gathered}
\boldsymbol{F}\left(\boldsymbol{x}_{1}^{*}\right)=\boldsymbol{F}\left(\boldsymbol{x}_{2}^{*}\right)=\ldots=\boldsymbol{F}\left(\boldsymbol{x}_{k}^{*}\right)=\boldsymbol{p}^{*}, \\
g\left(\boldsymbol{w}, \boldsymbol{x}_{1}^{*}\right)=g\left(\boldsymbol{w}, \boldsymbol{x}_{2}^{*}\right)=\ldots=g\left(\boldsymbol{w}, \boldsymbol{x}_{k}^{*}\right)=0, \\
\text { s.t. } \forall \boldsymbol{x}_{i}^{*}, \boldsymbol{x}_{j}^{*}, \boldsymbol{x}_{i}^{*} \neq \boldsymbol{x}_{j}^{*} \text { if } i \neq j .
\end{gathered}
$$


According to the above equations, for each weight vector $\boldsymbol{w}$, Eq. (4) is a multi-modal single-objective optimization problem with $k$ global optimal solutions. In this way, the original MMOP is decomposed into a set of multi-modal single-objective optimization sub-problems.

$$
\min _{\boldsymbol{x}} g(\boldsymbol{w}, \boldsymbol{x}) .
$$

Any multi-modal single-objective optimization algorithm can be used to optimize Eq. (4). In our proposed algorithm, we apply a greedy iterative algorithm to optimize it. The detailed implementation of the proposed MMEA will be discussed in the next section.

Our proposed algorithm uses a similar sub-population framework to the above mentioned algorithms in Section II-B In our algorithm, each weight vector has a sub-population containing the same number of solutions. In real-world applications, it is challenging to specify the sub-population size in prior since usually the number of equivalent Pareto subsets is unknown. In Section IV-B3, we will further examine the performance of our proposed MMEA with different specifications of the sub-population size.

\section{B. Implementation}

Algorithm 1 shows the framework of our decomposition based MMEA called MOEA/D-MM (MOEA/D for Multimodal Multi-objective optimization). At the beginning, $\lambda=$ $\lfloor N / \mu\rfloor$ weight vectors are generated with the same method as MOEA/D. In line 3, $\mu$ solutions are randomly assigned to each weight vector. With this settings, each weight vector can preserve at most $\mu$ equivalent solutions. For convenience, solutions assigned to $\boldsymbol{w}_{i}$ are denoted as $P_{i}$.

In each iteration, every sub-population is updated based on the $(\mu+1)$ scenario. For a sub-population $P_{i}$, an offspring solution is generated by the procedures described in Algorithm 2 Firstly, we randomly select a solution from $P_{i}$ as the first parent. To generate more diverse offspring solutions, the second parent is randomly selected from the union of neighborhood weight vectors' sub-populations. Then the generated offspring is added to $P_{i}$. In line 14, a solution is removed from $P_{i}$ based on the environmental selection procedure described in Algorithm 4

In the environmental selection process, the clearing [15] method is introduced to create a niching structure in the decision space. The main idea of clearing is that within a given clearing radius $\sigma$, the best solution takes all resources, i.e., other solutions will be removed. In MOEA/D-MM, the clearing radius is estimated using Algorithm 3 Before updating any sub-population, the clearing radius is set to the average Euclidean distance from each solution in the whole population to its $L$-th nearest neighbor in the decision space. In this paper, $L$ is set to $\lfloor N / 10\rfloor$.

Unlike the original clearing method, MOEA/D-MM only applies the clearing once in order to keep the sub-population size unchanged. In line 2 of Algorithm 4, a pair of points
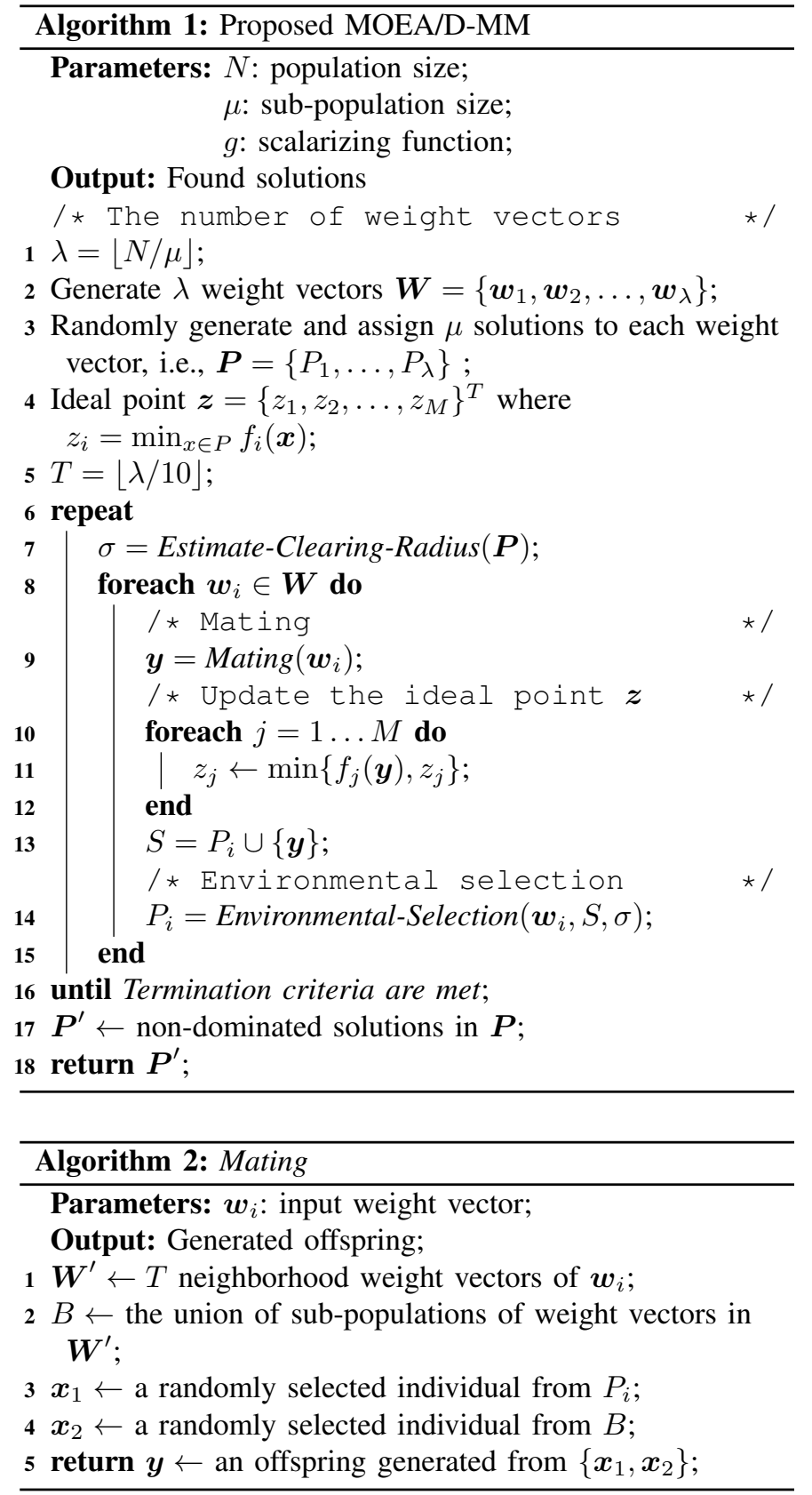

with the smallest distance between them in the current subpopulation are found. If the Euclidean distance between them in the decision space is smaller than the clearing radius, the one with the better scalarizing function value will survive. Otherwise, the solution with the worst scalarizing function value in the current sub-population is removed.

\section{Effectiveness of MOEA/D-MM}

In this section, we give some examples to illustrate the environmental selection mechanism in MOEA/D-MM. Fig. 3 shows a multi-polygon test problem [16] with four equivalent Pareto subsets. In this test problem, any solution inside the four hexagons (including solutions on the boundaries) is Pareto optimal. We assume that the sub-population size of each weight 

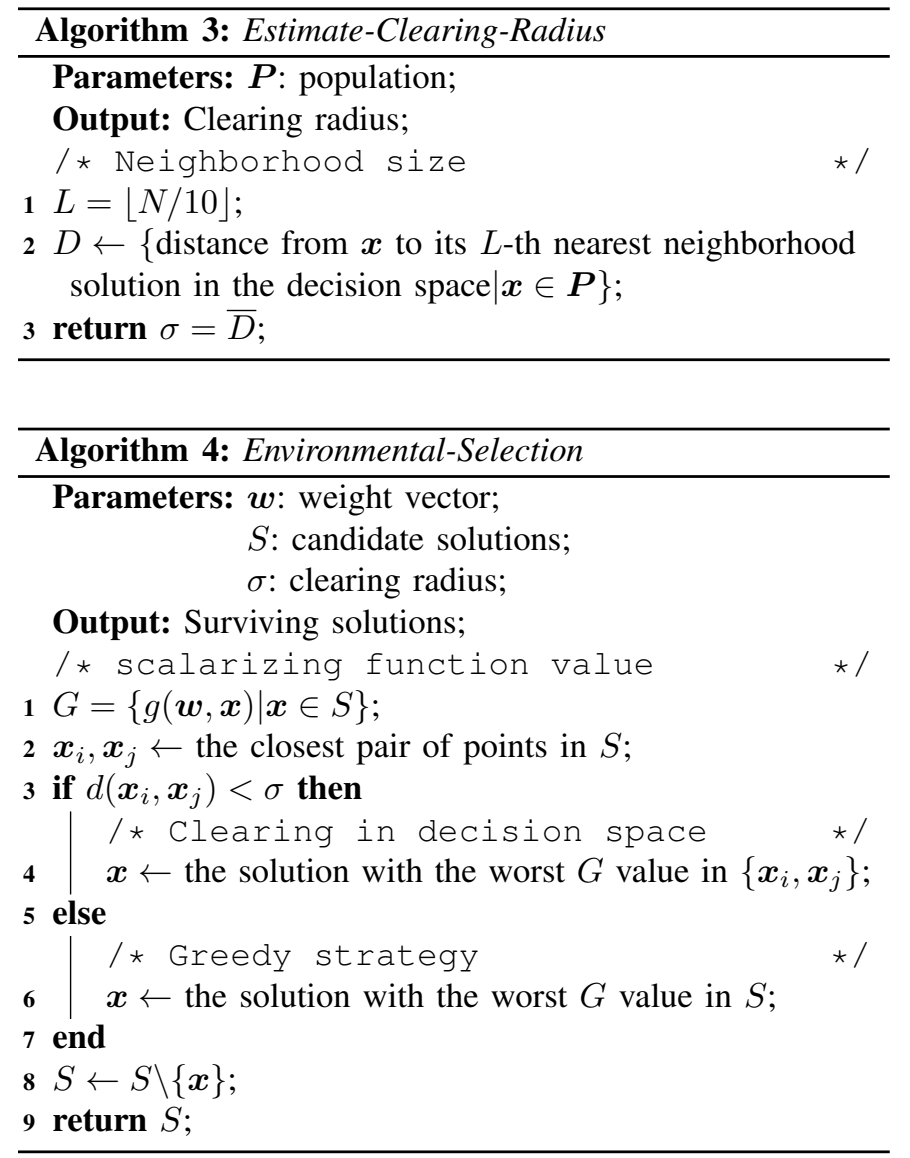

vector for MOEA/D-MM is $\mu=4$. For clarity, we focus on the sub-population of the weight vector $\boldsymbol{w}$ whose scalarizing function value is minimized at the center of each hexagon. Therefore, solutions in this sub-population are searching toward the four equivalent Pareto optimal solutions located at the centers of the hexagons. In each generation, an offspring is generated and added to the sub-population of $\boldsymbol{w}$, and a solution in this sub-population is removed. In each figure in Fig. 3 . one of the five solutions (denoted by $(A, B, C, D, E)$ ) will be removed by the environmental selection mechanism. The dashed circle(s) in each figure represent the clearing radius.

In Fig. 3 (a), the distance between solutions $A$ and $B$ is smaller than the clearing radius $\sigma$. Therefore, $A$ is removed since it is worse than $B$. In some cases, the clearing does not remove any solution since all solutions in the current subpopulation are not close to each other. Then the environmental selection is based on the greedy removal strategy, i.e., remove the worst solution in the current sub-population. For example, in Fig. 3 (b), solution $C$ is removed. However, the greedy removal strategy cannot always make the best decision. In Fig. 3 (c), solutions $D$ and $E$ are searching toward the same Pareto optimal solution in the right bottom hexagon. Although one of them is expected to be removed, the clearing mechanism does not work because the distance between solutions $D$ and $E$ is larger than $\sigma$. As opposed to our expectation, a potentially good solution $C$ is removed. Although the greedy strategy may make some mistakes, the clearing method can recover from the situation that several solutions in the same sub-population converge to the same Pareto optimal solution. After a number of iterations, the distance between solutions $D$ and $E$ will eventually become smaller than $\sigma$, then one of them will be removed. In Fig. 3 (d), solution $D$ will be removed by the clearing mechanism. Notice that solution $C$ is Fig. 3 (d) is a newly generated solution. With the clearing mechanism and the greedy removal strategy, MOEA/D-MM can efficiently preserve equivalent Pareto optimal solutions in sub-populations.

\section{EXPerimental StUdies}

\section{A. Experimental settings}

1) Test problems: The following four MMOPs are used to benchmark the performance of MMEAs: the SYM-PART problems [17], the SSUF1 and SUF3 test problems [4] and the multi-polygon test problems [16]. Similar to [11], we use the multi-polygon test problems to test the scalability of MOEA/D-MM regarding the dimension of the decision space. Parameters of the selected test problems are listed in Table [1] In this table, $x_{i}$ denotes the $i$ th decision variable, and the dimension of the objective space and the decision space of each test problem are denoted by $M$ and $D$, respectively. For the SYM-PART test problem, the length of each Pareto subset is $2 a$, and the vertical and horizontal distances between the centers of two adjacent Pareto subsets are specified by $b$ and $c$, respectively.

2) Performance indicators: In our experiments, we use the modified inverted generational distance $\left(\mathrm{IGD}^{+}\right)[18]$ and the IGDX [19] indicators for performance comparison. These two indicators are used to assess the quality of the obtained solution set in the objective and decision space, respectively. The $\mathrm{IGD}^{+}$indicator improves the original IGD [20] indicator by using a special distance function. In contrast to the original IGD indicator, the $\mathrm{IGD}^{+}$indicator is weakly Pareto compliant. Formally, given a reference point set $P$ in the objective space, the $\mathrm{IGD}^{+}$value of a set $A$ can be calculated using Eq. (5).

$$
\begin{gathered}
d^{+}(\boldsymbol{z}, \boldsymbol{a})=\sqrt{\sum_{i=1}^{m}\left(\max \left\{a_{i}-z_{i}, 0\right\}\right)^{2}}, \\
\operatorname{IGD}^{+}(A)=\frac{1}{|P|} \sum_{\boldsymbol{p} \in P} \min \left\{d^{+}(\boldsymbol{p}, \boldsymbol{a}) \mid \forall \boldsymbol{a} \in A\right\} .
\end{gathered}
$$

The IGDX value of a set $A$ for a reference point set $S$ in the decision space is given by Eq. 6.

$$
\operatorname{IGDX}(A)=\frac{1}{|S|} \sum_{\boldsymbol{x} \in S} \min \{d(\boldsymbol{x}, \boldsymbol{a}) \mid \forall \boldsymbol{a} \in A\},
$$

where $d$ is the Euclidean distance function.

For the IGDX indicator, the reference point set $S$ is generated by uniformly sampling 10,000 points on the Pareto sets of each test problem. Then, the image of $S$ in the objective space (i.e., $P$ ) is used to calculate the $\mathrm{IGD}^{+}$indicator. 


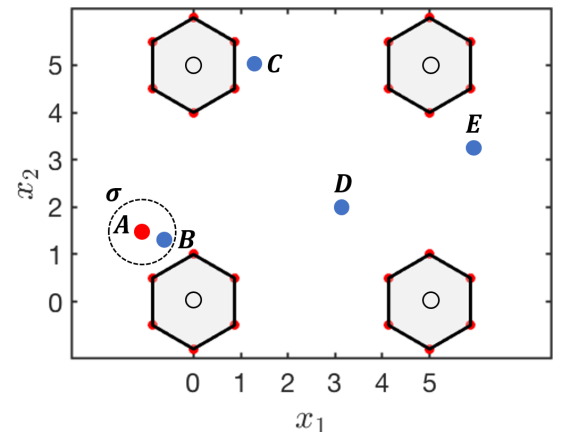

(a) $A$ is removed.

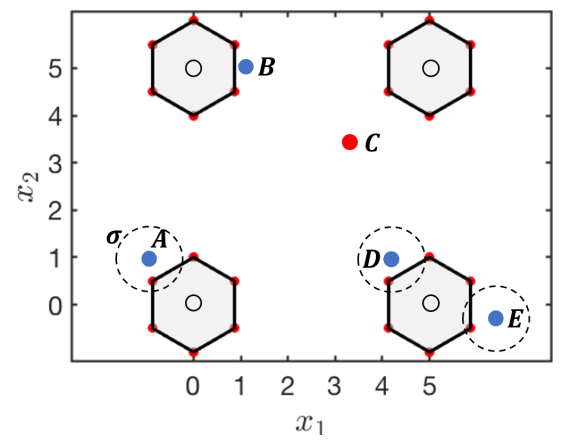

(c) $C$ is removed.

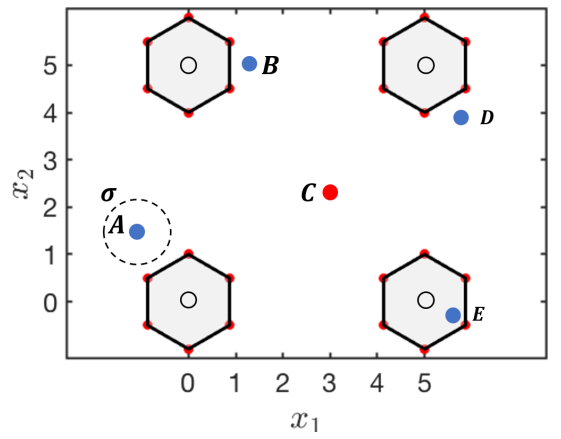

(b) $C$ is removed.

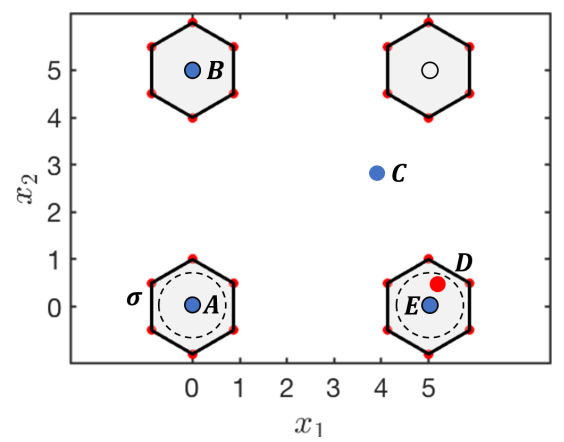

(d) $D$ is removed.

Fig. 3. Illustration of the effect of the environmental selection mechanism of MOEA/D-MM on multi-polygon test problem.

TABle i. Parameter Settings For Selected Test Problems.

\begin{tabular}{cccccc}
\hline & \multicolumn{5}{c}{ Parameters } \\
\cline { 2 - 6 } Problems & $M$ & $D$ & Search Space & Special Parameters & Number of Pareto subsets \\
\hline SYM-PART & 2 & 2 & $x_{i} \in[-100,100]^{2}$ & $a=2, b=10, c=10$ & 9 \\
SSUF1 & 2 & 2 & $x_{1} \in[1,3]$ and $x_{2} \in[-1,1]$ & - & 2 \\
SUF3 & 2 & 2 & $x_{1} \in[0,1]$ and $x_{2} \in[1,2]$ & - & 2 \\
Multi-polygon & 6 & $\{2,4,6,8,10\}$ & $x_{i} \in[-100,100]^{D}$ & centers: $(0,0),(0,5),(5,0),(5,5)$ & 4 \\
\hline
\end{tabular}

3) Selected algorithms: To verify the effectiveness of MOEA/D-MM on solving MMOPs, we compare its performance with the original MOEA/D with Tchebycheff and PBI scalarizing functions as well as three recentlyproposed MMEAs: MOEA/D-AD [8], DNEA [9] and MO_Ring_PSO_SCD [10]. All algorithms are implemented on the PlatEMO [21] platform. Parameters for each algorithm are set to the suggested values in the original paper. In our experiments, each algorithm is evaluated with population size 300 and 100, 000 evaluations. All algorithms are examined on each test problem for 31 times in order to obtain reliable comparison results. For each algorithm on each test problem, a single run with the median $\mathrm{HV}$ over the 31 runs is selected for visualization.

\section{B. Experimental results}

1) Comparison with MOEA/D: In this section, we compare the performance of MOEA/D-MM and MOEA/D on the SUF3 test problem. Both of them use the Tchebycheff scalarizing function. In Fig. 4, most solutions obtained by MOEA/D are located in one of the two Pareto subsets. However, MOEA/DMM successfully locates all Pareto subsets. The simulation results clearly show that MOEA/D-MM can effectively preserve equivalent Pareto optimal solutions with the sub-population framework.

2) Benchmark results on MMOPs: In this section we specify the sub-population size as 4 in MOEA/D-MM. This specification is further discussed in the next section.

Figs. 5 and 6 visualize the non-dominated solutions in the final population of MOEA/D-MM with Tchebycheff (MOEA/D$\mathrm{MM}-\mathrm{TCH}$ ) and PBI (MOEA/D-MM-PBI) scalarizing functions in the decision space on each test problem, respectively. The black lines in Figs. 5 (a)-(c) and Figs. 6(a)-(c) represent the Pareto set of the corresponding test problem. In Figs. 5 (d) and 6(d), the four hexagons are equivalent Pareto subsets. From the visualization results, we can see that MOEA/D-MM is able to obtain solution sets with very good coverage on all Pareto subsets. 


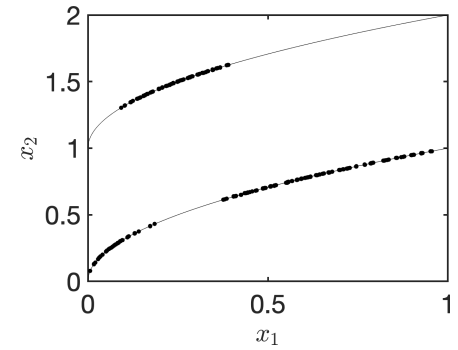

(a) MOEA/D

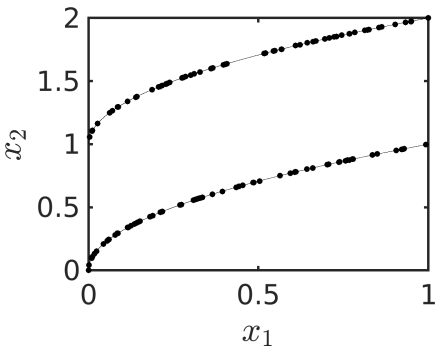

(b) MOEA/D-MM
Fig. 4. Comparison of MOEA/D-MM and MOEA/D on the SUF3 test problem. In each figure, the thin lines denote the equivalent Pareto subsets, and the dark circles represent the obtained solutions.

Tables III and III present the numerical comparison results among the seven algorithms. In each table, we use the Wilconxon rank-sum test with $p<0.05$ to compare each algorithm with MOEA/D-MM-TCH. The symbols,$+ \approx$ and indicate that the corresponding algorithm is significantly better than, no significant difference from, and significantly worse than the performance of MOEA/D-MM-TCH, respectively. Best results in each table are highlighted.

As shown in Table [II. MOEA/D-MM-TCH has the best performance among the tested algorithms regarding the IGDX indicator, especially on the multi-polygon test problems with higher dimensional decision spaces, e.g. $D=4,6,8,10$. Notice that MOEA/D-MM and MOEA/D with the PBI function perform worse than the Tchebycheff function almost on all test problems. Numerical results indicate that the Tchebycheff function can obtain more uniform solutions than the PBI function on selected test problems. The main reason is that these four test problems are all convex test problems. The PBI scalarizing function does not work well on such kind of problems. In particular, MO_Ring_PSO_SCD performs the best on the SUF3, SSUF1 test problems and DNEA outperforms others on the SYM-PART test problem.

Table III shows the average $\mathrm{IGD}^{+}$indicator value of each algorithm on each test problem. As observed in the literature [4] [8], the IGD values of MMEAs are usually worse than MOEAs. This is because equivalent solutions are the same in the objective space, which means that they do not contribute to the IGD indicator. Since $\mathrm{IGD}^{+}$is similar to IGD, MMEAs are expected to have worse $\mathrm{IGD}^{+}$indicator values than MOEAs. Among all algorithms, MOEA/D with the Tchebycheff function achieves the best performance with respect to the $\mathrm{IGD}^{+}$ indicator. Among tested MMEAs, MO_Ring_PSO_SCD and DNEA have the best performance on MMOPs with the 2dimensional decision space while MOEA/D-MM-TCH outperforms other MMEAs with higher dimensional decision spaces in terms of the $\mathrm{IGD}^{+}$indicator.

3) Influence of sub-population size: In this section, we investigate the influence of the sub-population size on the performance of MOEA/D-MM. In MOEA/D-MM, the subpopulation size determines the maximum number of equivalent solutions that a weight vector can preserve. Suppose we are handling an MMOP with $k$ equivalent Pareto subsets, and the sub-population size is $\mu$. Since the population size is $N$, the number of weight vectors is $\lfloor N / \mu\rfloor$. When $\mu=k$, all equivalent solutions can be covered. When $\mu<k$, each weight vector can only preserve at most $\mu$ equivalent solutions. In this case, the decision maker has less choices when selecting a final solution. However, MOEA/D-MM may have a better search ability in the objective space since more weight vectors are used. When $\mu>k$, the performance of MOEA/D-MM will be deteriorated mainly due to the following two reasons. Firstly, the maximum number of equivalent solutions in each subpopulation is not changed, which means that some solutions in the sub-population of each weight vector may have no contribution to the quality of the solution set. Secondly, a larger sub-population size means that less weight vectors are used, which will also reduce the search ability.

Fig. 7 shows the average IGDX and $\mathrm{IGD}^{+}$indicator values obtained by MOEA/D-MM-TCH on the multi-polygon test problems with four equivalent Pareto subsets. Five specifications of $\mu(\mu=2,3,4,5,6)$ are examined for the multipolygon test problems in five decision spaces ( $D$-dimensional decision space for $D=2,4,6,8,10)$. In Fig. 8 , we show the final populations of MOEA/D-MM-TCH on the polygon test problem with two-dimensional decision space for two settings of $\mu: \mu=2$ and $\mu=6$. From Fig. 7 (a), when $\mu<4$, IGDX values do not change too much. Because the IGDX indicator only measures the coverage of the Pareto set instead of the number of equivalent solutions. As shown in Fig. 8 (a), when $\mu=2$, although a weight vector can only preserve two equivalent solutions, the coverage of Pareto subsets are almost the same as Fig. 5 (d). This is because the number of weight vectors is two times as large as in the case of $\mu=4$. According to Fig. 7 (b), when $\mu>4$, the performance of the algorithm degrades in terms of the IGDX and IGD ${ }^{+}$ indicators. In Fig. 8 (b), we can also observe that compared to $\mu=2$ and $\mu=4$, fewer non-dominated solutions are obtained when $\mu=6$ although all Pareto subsets are covered. The experimental results indicate that a smaller sub-population size is preferred in MOEA/D-MM.

\section{CONCluding Remarks}

In this paper, we proposed MOEA/D-MM, a simple yet efficient multi-modal multi-objective optimization algorithm based on MOEA/D. We introduce a clearing mechanism and a greedy removal strategy to MOEA/D with the sub-population framework. The proposed algorithm shows promising performance in comparison with recently-proposed MMEAs on various MMOPs, especially on large-scale test problems.

Several interesting research topics are left for future work. For example, a dynamic sub-population size adjustment strategy is needed for the handling of real-world optimization problems without knowledge about the number of Pareto sets. The development of new test problems and new performance indicators in the decision space is also an interesting research topic in the filed of multi-modal multi-objective optimization. 


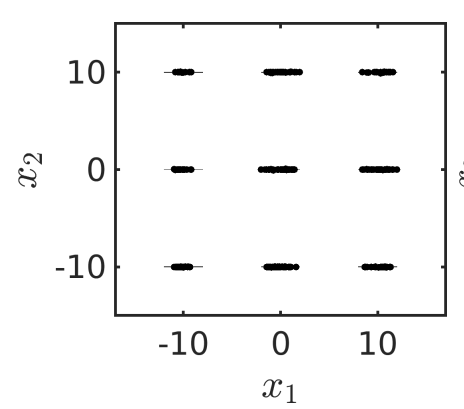

(a) SYM-PART.

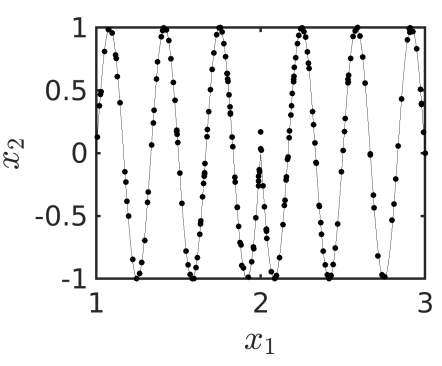

(b) SSUF1.

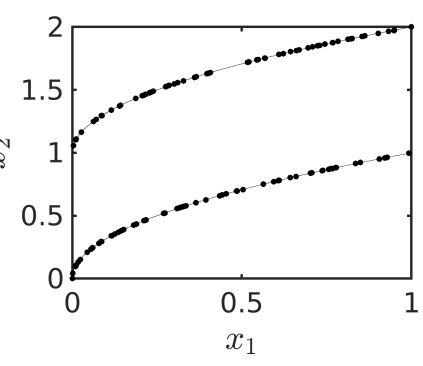

(c) SUF3.

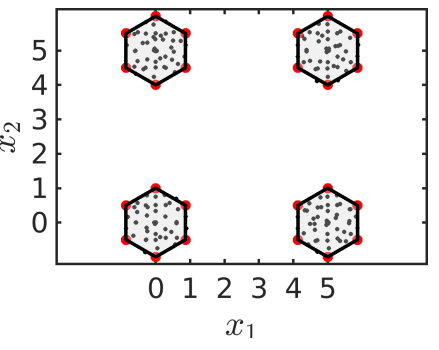

(d) $\operatorname{Multi-polygon}(D=2)$.

Fig. 5. Visualization of non-dominated solutions in the final population of MOEA/D-MM with the Tchebycheff function in the decision space on each test problem.

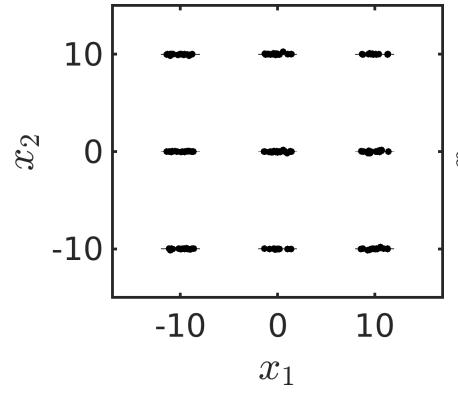

(a) SYM-PART.

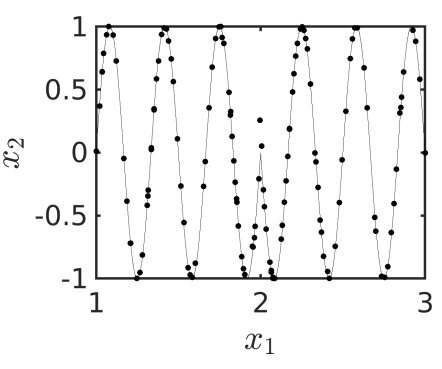

(b) SSUF1.

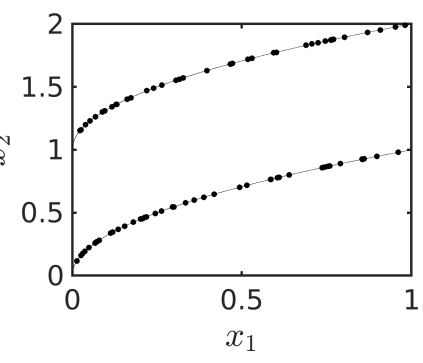

(c) SUF3.

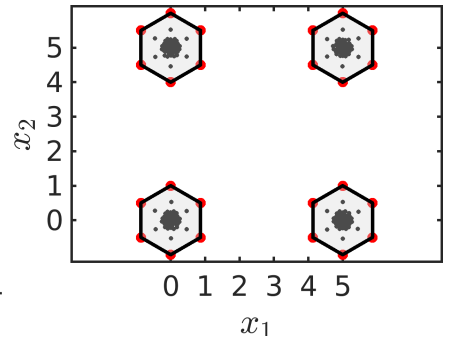

(d) $\operatorname{Multi-polygon}(D=2)$.

Fig. 6. Visualization of non-dominated solutions in the final population obtained by MOEA/D-MM with the PBI function in the decision space on each test problem.

TABLE II. Average IGDX VALUeS OVer 31 RUnS. Best RESUlts ARE Highlighted.

\begin{tabular}{|c|c|c|c|c|c|c|c|c|c|}
\hline \multirow[b]{2}{*}{ Problems } & \multirow[b]{2}{*}{$M$} & \multirow[b]{2}{*}{$D$} & \multicolumn{2}{|c|}{ MOEA/D-MM } & \multirow[b]{2}{*}{ MO_Ring_PSO_SCD } & \multirow[b]{2}{*}{ MOEA/D-AD } & \multirow[b]{2}{*}{ DNEA } & \multicolumn{2}{|c|}{ MOEA/D } \\
\hline & & & $\mathrm{TCH}$ & PBI & & & & $\mathrm{TCH}$ & PBI \\
\hline SUF3 & 2 & 2 & $1.2359 \mathrm{e}-2$ & $2.0712 \mathrm{e}-2-$ & $7.3534 \mathrm{e}-3+$ & $1.2853 \mathrm{e}-2 \approx$ & $1.2541 \mathrm{e}-2 \approx$ & $2.1853 \mathrm{e}-1-$ & $2.5902 \mathrm{e}-1-$ \\
\hline SSUF1 & 2 & 2 & $4.2628 \mathrm{e}-2$ & $6.0374 \mathrm{e}-2-$ & $3.3075 \mathrm{e}-2+$ & $4.8514 \mathrm{e}-2-$ & $3.5394 \mathrm{e}-2+$ & $1.3177 \mathrm{e}-1-$ & $1.4571 \mathrm{e}-1-$ \\
\hline SYM-PART & 2 & 2 & $1.5503 \mathrm{e}-1$ & $1.9154 \mathrm{e}-1-$ & $1.1212 \mathrm{e}-1+$ & $5.9621 \mathrm{e}-2+$ & $5.7180 \mathrm{e}-1-$ & $1.8686 \mathrm{e}+0-$ & $9.7947 \mathrm{e}-1-$ \\
\hline \multirow{6}{*}{ Multi-polygon } & 6 & 2 & $1.0902 \mathrm{e}-1$ & $2.1422 \mathrm{e}-1-$ & $1.1658 \mathrm{e}-1-$ & $1.7744 \mathrm{e}-1-$ & $1.0430 \mathrm{e}-1+$ & $1.7188 \mathrm{e}-1-$ & $2.4330 \mathrm{e}-1-$ \\
\hline & 6 & 4 & $2.4670 \mathrm{e}-1$ & $3.7535 \mathrm{e}-1-$ & $3.8229 \mathrm{e}-1-$ & $2.9541 \mathrm{e}-1-$ & $3.7152 \mathrm{e}+0-$ & $4.4101 \mathrm{e}+0-$ & $5.2624 \mathrm{e}+0-$ \\
\hline & 6 & 6 & $4.5473 \mathrm{e}-1$ & $5.9612 \mathrm{e}-1-$ & $1.0294 \mathrm{e}+0-$ & $5.0676 \mathrm{e}-1-$ & $6.5989 \mathrm{e}+0-$ & $6.4156 \mathrm{e}+0-$ & $7.0966 \mathrm{e}+0-$ \\
\hline & 6 & 8 & $7.3573 e-1$ & $8.3894 \mathrm{e}-1-$ & $2.8267 \mathrm{e}+0-$ & $8.9567 \mathrm{e}-1-$ & $7.8238 \mathrm{e}+0-$ & $7.5401 \mathrm{e}+0-$ & $8.3309 \mathrm{e}+0-$ \\
\hline & 6 & 10 & $1.1849 \mathrm{e}+0$ & $1.1283 \mathrm{e}+0 \approx$ & $6.4177 \mathrm{e}+0-$ & $2.5597 \mathrm{e}+0-$ & $8.9284 \mathrm{e}+0-$ & $8.5160 \mathrm{e}+0-$ & $9.4045 \mathrm{e}+0-$ \\
\hline & 6 & 100 & $3.0347 \mathrm{e}+1$ & $3.2792 \mathrm{e}+1-$ & $2.9945 \mathrm{e}+2-$ & $8.0263 \mathrm{e}+1-$ & $2.9788 \mathrm{e}+1+$ & $3.0377 \mathrm{e}+1-$ & $3.1208 \mathrm{e}+1 \approx$ \\
\hline \multicolumn{3}{|c|}{$+/-/ \approx$} & Baseline & $0 / 8 / 1$ & $3 / 6 / 0$ & $1 / 7 / 1$ & $3 / 5 / 1$ & $0 / 9 / 0$ & $0 / 8 / 1$ \\
\hline
\end{tabular}

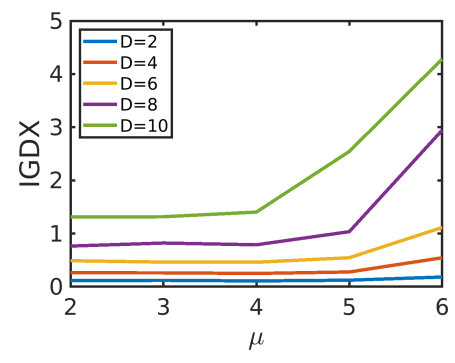

(a) IGDX versus $\mu$.

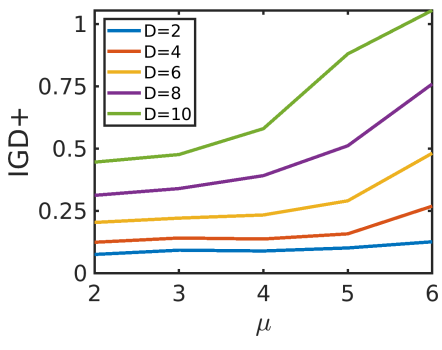

(b) $\mathrm{IGD}^{+}$versus $\mu$.

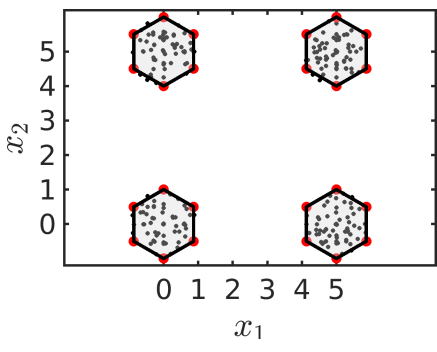

(a) $\mu=2$

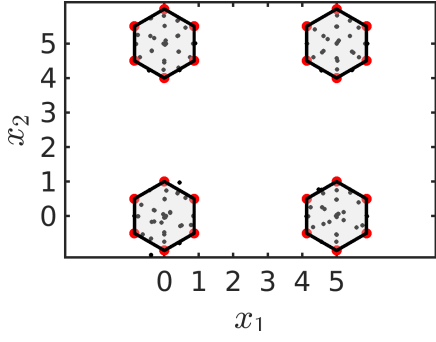

(b) $\mu=6$

Fig. 7. The average of IGDX and IGD ${ }^{+}$indicator values with respect to sub-population size $\mu$.

Fig. 8. The final populations of MOEA/D-MM-TCH with two settings of $\mu: \mu=2$ and $\mu=6$. 
TABLE III. AVERAGE IGD ${ }^{+}$VALUES OVER 31 RUNS. BEST RESUltS ARE HIGHLIGHTED.

\begin{tabular}{|c|c|c|c|c|c|c|c|c|c|}
\hline \multirow[b]{2}{*}{ Problems } & \multirow[b]{2}{*}{$M$} & \multirow[b]{2}{*}{$D$} & \multicolumn{2}{|c|}{ MOEA/D-MM } & \multirow[b]{2}{*}{ MO_Ring_PSO_SCD } & \multirow[b]{2}{*}{ MOEA/D-AD } & \multirow[b]{2}{*}{ DNEA } & \multicolumn{2}{|c|}{ MOEA/D } \\
\hline & & & $\mathrm{TCH}$ & PBI & & & & $\mathrm{TCH}$ & PBI \\
\hline SUF3 & 2 & 2 & $4.1228 \mathrm{e}-3$ & $6.5426 \mathrm{e}-3-$ & $2.8444 \mathrm{e}-3+$ & $5.0655 \mathrm{e}-3-$ & $1.8247 \mathrm{e}-3+$ & $2.3882 \mathrm{e}-3+$ & $8.4060 \mathrm{e}-3 \approx$ \\
\hline SSUF1 & 2 & 2 & $2.4583 \mathrm{e}-3$ & $3.2304 \mathrm{e}-3-$ & $1.6090 \mathrm{e}-3+$ & $2.9579 \mathrm{e}-3-$ & $1.4054 \mathrm{e}-3+$ & $8.5769 \mathrm{e}-4+$ & $1.0541 \mathrm{e}-3+$ \\
\hline SYM-PART & 2 & 2 & $4.2798 \mathrm{e}-2$ & $8.9186 \mathrm{e}-2-$ & $2.3064 \mathrm{e}-2+$ & $1.7412 \mathrm{e}-2+$ & $1.0714 \mathrm{e}-2+$ & $1.3249 \mathrm{e}-2+$ & $7.3563 \mathrm{e}-2-$ \\
\hline \multirow{6}{*}{ Multi-polygon } & 6 & 2 & $9.0390 \mathrm{e}-2$ & $1.8361 \mathrm{e}-1-$ & $6.6848 \mathrm{e}-2+$ & $1.6209 \mathrm{e}-1-$ & $5.0966 \mathrm{e}-2+$ & $7.1261 \mathrm{e}-2+$ & $1.3933 \mathrm{e}-1-$ \\
\hline & 6 & 4 & $1.3741 \mathrm{e}-1$ & $2.6096 \mathrm{e}-1-$ & $1.9312 \mathrm{e}-1-$ & $2.2258 \mathrm{e}-1-$ & $1.4665 \mathrm{e}-1-$ & $1.1396 \mathrm{e}-1+$ & $2.5126 \mathrm{e}-1-$ \\
\hline & 6 & 6 & $2.2770 \mathrm{e}-1$ & $3.9712 \mathrm{e}-1-$ & $6.0390 \mathrm{e}-1-$ & $3.1566 \mathrm{e}-1-$ & $3.0919 \mathrm{e}-1-$ & $1.6600 \mathrm{e}-1+$ & $4.7236 \mathrm{e}-1-$ \\
\hline & 6 & 8 & $3.5604 \mathrm{e}-1$ & $5.7543 e-1-$ & $1.6849 \mathrm{e}+0-$ & $4.8743 \mathrm{e}-1-$ & $4.7776 \mathrm{e}-1-$ & $2.3664 \mathrm{e}-1+$ & $6.6033 \mathrm{e}-1-$ \\
\hline & 6 & 10 & $5.0716 \mathrm{e}-1$ & 7.2597e-1 - & $4.1616 \mathrm{e}+0-$ & $7.1944 \mathrm{e}-1-$ & $6.3330 \mathrm{e}-1-$ & $3.0650 \mathrm{e}-1+$ & $8.2931 \mathrm{e}-1-$ \\
\hline & 6 & 100 & $1.7306 \mathrm{e}+1$ & $1.4175 \mathrm{e}+1+$ & $7.0584 \mathrm{e}+2-$ & $1.6153 \mathrm{e}+2-$ & $8.1872 \mathrm{e}+0+$ & $3.5356 \mathrm{e}+0+$ & $4.1586 \mathrm{e}+0+$ \\
\hline \multicolumn{3}{|c|}{$+/-/ \approx$} & Baseline & $1 / 8 / 0$ & $4 / 5 / 0$ & $1 / 8 / 0$ & $5 / 4 / 0$ & $9 / 0 / 0$ & $2 / 6 / 1$ \\
\hline
\end{tabular}

\section{REFERENCES}

[1] K. Deb, A. Pratap, S. Agarwal, and T. Meyarivan, "A fast and elitist multiobjective genetic algorithm: NSGA-II," IEEE Transactions on Evolutionary Computation, vol. 6, no. 2, pp. 182-197, April 2002.

[2] Q. Zhang and H. Li, "MOEA/D: a multiobjective evolutionary algorithm based on decomposition," IEEE Transactions on Evolutionary Computation, vol. 11, no. 6, pp. 712-731, December 2007.

[3] A. Moshaiov, "The paradox of multimodal optimization: concepts vs. species in single and multi-objective problems," in Proc. of 2016 IEEE Congress on Evolutionary Computation, Vancouver, BC, Canada, July 24-29 2016, pp. 1743-1748.

[4] J. J. Liang, C. T. Yue, and B. Y. Qu, "Multimodal multi-objective optimization: a preliminary study," in Proc. of 2016 IEEE Congress on Evolutionary Computation, Vancouver, BC, Canada, July 24-29 2016 , pp. 2454-2461.

[5] O. Schütze, M. Vasile, and C. A. Coello Coello, "Computing the set of epsilon-efficient solutions in multiobjective space mission design," Journal of Aerospace Computing, Information, and Communication, vol. 8, no. 3, pp. 53-70, March 2011.

[6] A. Jaszkiewicz, "On the performance of multiple-objective genetic local search on the 0/1 knapsack problem - a comparative experiment," IEEE Transactions on Evolutionary Computation, vol. 6, no. 4, pp. 402-412, August 2002.

[7] K. Deb, Multi-objective optimization using evolutionary algorithms. USA: John Wiley \& Sons, Inc., July 2001.

[8] R. Tanabe and H. Ishibuchi, "A decomposition-based evolutionary algorithm for multi-modal multi-objective optimization," in Proc. of Parallel Problem Solving from Nature - PPSN XV, Coimbra, Portugal, September 8-12 2018, pp. 249-261.

[9] Y. Liu, H. Ishibuchi, Y. Nojima, N. Masuyama, and K. Shang, "A double-niched evolutionary algorithm and its behavior on polygon-based problems," in Proc. of Parallel Problem Solving from Nature - PPSN XV, Coimbra, Portugal, September 8-12 2018, pp. 262-273.

[10] C. Yue, B. Qu, and J. Liang, "A multiobjective particle swarm optimizer using ring topology for solving multimodal multiobjective problems," IEEE Transactions on Evolutionary Computation, vol. 22, no. 5, pp. 805-817, October 2018.

[11] Y. Peng, H. Ishibuchi, and K. Shang, "Multi-modal multi-objective optimization: problem analysis and case studies," in Proc. of IEEE Sym- posium Series on Computational Intelligence, Xiamen, China, December 6-9 2019, pp. 1865-1872.

[12] Y. Qi, X. Ma, F. Liu, L. Jiao, J. Sun, and J. Wu, "MOEA/D with adaptive weight adjustment," Evolutionary Computation, vol. 22, no. 2, pp. 231264, June 2014.

[13] H. Liu, F. Gu, and Q. Zhang, "Decomposition of a multiobjective optimization problem into a number of simple multiobjective subproblems," IEEE Transactions on Evolutionary Computation, vol. 18, no. 3, pp. 450-455, June 2014.

[14] C. Hu and H. Ishibuchi, "Incorporation of a decision space diversity maintenance mechanism into MOEA/D for multi-modal multi-objective optimization," in Proc. of Genetic and Evolutionary Computation Conference Companion, Kyoto, Japan, July 15-19 2018, pp. 1898-1901.

[15] A. Petrowski, "A clearing procedure as a niching method for genetic algorithms," in Proc. of IEEE International Conference on Evolutionary Computation, Nagoya, Japan, May 20-22 1996, pp. 798-803.

[16] H. Ishibuchi and Y. Peng, "A scalable multimodal multiobjective test problem," in Proc. of 2019 IEEE Congress on Evolutionary Computation, Wellington, New Zealand, June 10-13 2019, pp. 302-309.

[17] G. Rudolph, B. Naujoks, and M. Preuss, "Capabilities of EMOA to detect and preserve equivalent Pareto subsets," in Proc. of Evolutionary Multi-Criterion Optimization, Matsushima, Japan, March 5-8 2007, pp. 36-50.

[18] H. Ishibuchi, H. Masuda, Y. Tanigaki, and Y. Nojima, "Modified distance calculation in generational distance and inverted generational distance," in Proc. of Evolutionary Multi-Criterion Optimization, Guimares, Portugal, March 29-April 1 2015, pp. 110-125.

[19] A. Zhou, Q. Zhang, and Y. Jin, "Approximating the set of Pareto-optimal solutions in both the decision and objective spaces by an estimation of distribution algorithm," IEEE Transactions on Evolutionary Computation, vol. 13, no. 5, pp. 1167-1189, October 2009.

[20] C. A. Coello Coello and M. Reyes Sierra, "A study of the parallelization of a coevolutionary multi-objective evolutionary algorithm," in Proc. of MICAI 2004: Advances in Artificial Intelligence, Mexico City, Mexico, April 26-30 2004, pp. 688-697.

[21] Y. Tian, R. Cheng, X. Zhang, and Y. Jin, "PlatEMO: A MATLAB platform for evolutionary multi-objective optimization," IEEE Computational Intelligence Magazine, vol. 12, no. 4, pp. 73-87, November 2017. 